\title{
Modélisation du transport solide en réseau d'assainissement unitaire : le modèle HYPOCRAS
}

\author{
Jean-Luc Bertrand-Krajewski \\ CIRSEE - Lyonnaise des Eaux-Dumez \\ 38 rue du Président Wilson, 78230 Le Pecq
}

\section{Introduction}

Depuis le milieu des années soixante-dix, la pollution des eaux pluviales véhiculées par les réseaux d'assainissement est devenue une préoccupation importante, et constitue aujourd hui un champ d'investigation majeur en hydrologie urbaine. Les solides entraînés par le ruissellement et transportés au sein des réseaux d'assainissement jouent un rôle particulier dans ce contexte, avec des conséquences multiples: encrassement des avaloirs et des collecteurs, rejets directs au milieu naturel en réseaux pluviaux séparatifs ou par les déversoirs en réseaux unitaires, surcharges des stations d'épuration. L'impact de ces rejets sur le milieu naturel est important, aussi bien à court qu'à long terme, même s'il est encore difficile aujourd hui de le quantifier avec précision. Ces rejets de solides, déjà indésirables par eux-mêmes (ensablement des cours d'eau, consommation d'oxygène dissous, colmatage des frayères de poissons, etc.), sont d'autant plus graves que les particules solides constituent le support sur lequel sont adsorbés d'autres polluants, notamment les métaux lourds et les hydrocarbures (Wageningen 1986, 1989. IAWPRC 1989, Moffa 1990).
Pour tenter de réduire ces nuisances, diverses solutions ont été mises en œuvre :

- techniques compensatoires ou alternatives, visant à réduire ou limiter l'arrivée des solides et des polluants dans les réseaux: chaussées poreuses, réduction des ruissellements pluviaux, stockages intermédiaires, etc. (Baladès et al. 1991) ;

- meilleur entretien et gestion plus rigoureuse des avaloirs et des collecteurs ;

- création de bassins de pollution, de chambres de dessablement, de séparateurs.

Les études et recherches se poursuivent sur l'impact des eaux pluviales sur le milieu récepteur, domaine complexe où l'interdisciplinarité est de rigueur, et commencent à se développer sur le comportement des stations d'épuration en réponse aux débits et flux polluants en temps de pluie. D'autre part, des recherches sont menées sur les possibilités de traitement direct des eaux pluviales (décantabilité, possibilités de traitement physico-chimique, etc.).

Une meilleure connaissance des phénomènes se produisant à l'amont et au sein des réseaux d'assainissement est devenue indispensable. Elle passe par des investigations

\section{Solid transport modelling in combined sewer systems} The HYPOCRAS model

A global and conceptual model for solid production and transfer in combined sewer systems has been established. This model, named HYPOCRAS, is valid for small urban catchments of less than 100 ha. Its storm weather part simulates the sediment build-up over the catchment, the washoff by rainfall, the solid transfer along the sewer pipes, the erosion and sedimentation of deposits. Two classes of particles are distinguished: the fine ones $\left(d_{50}=40 \mu \mathrm{m}\right)$ which are transported in suspension and the coarse ones $\left(d_{50}=400 \mu \mathrm{m}\right)$ which constitute the deposits and which are transported in bed load or in suspension depending on the hydraulic conditions. The model has been calibrated and verified for three different catchments, with satisfactory results. 
importantes in situ, et par l'établissement de modèles numériques. Ainsi la modélisation du transport solide en réseau est intéressante à plusieurs titres :

- favoriser la connaissance et la compréhension des phénomènes ;

- mettre en évidence les paramètres les plus déterminants et leurs relations essentielles ;

- contribuer à un dimensionnement plus judicieux des ouvrages (collecteurs, bassins, etc.) en permettant leur simulation ;

- permettre, à plus long terme, une gestion en temps réel des réseaux ;

- donner une meilleure estimation de la quantité et de la qualité des flux polluants rejetés au milieu récepteur.

Plusieurs modèles de transport solide en réseau d'assainissement ont été élaborés depuis une vingtaine d'années, fondés sur des approches et des conceptions diverses. La qualité des résultats obtenus est très variable, en raison de l'extrême complexité des phénomènes mis en jeu et de la connaissance limitée que nous en avons : la modélisation du transport solide reste une question difficile (BertrandKrajewski 1991a).

A partir des connaissances acquises récemment sur les particules solides et leurs modes de transport en réseau (Laplace 1991, Chebbo et al. 1990), nous avons essayé d'établir un modèle destiné, dans une première étape, aux petits bassins versants urbains équipés de réseaux unitaires. Ce modèle, baptisé HYPOCRAS ( ${ }^{1}$ ) (Hydrogrammes et Pollutogrammes Calculés en Réseau d'Assainissement), permet de simuler les pollutogrammes et les variations des dépôts, tout en conservant une description simple du bassin versant et du réseau.

\section{Critères de choix pour une modélisation}

Les connaissances actuelles des phénomènes physiques gouvernant le transport solide en réseau d'assainissement sont encore limitées et parfois contradictoires. Dans ces conditions, un modèle conceptuel nous paraît plus judicieux qu'un modèle de type mécaniste, d'autant que nous ne disposons pas encore d'équations correctement validées dans ce domaine.

Pour chaque étape du processus du transport solide, les connaissances acquises sont de niveaux très variables. Introduire des équations complexes pour certaines étapes alors que d'autres ne sont connues que de manière très empirique et approximative serait une démarche incohérente. Au sein d'un modèle, il est préférable de tendre vers un niveau de complexité homogène pour l'ensemble des étapes du calcul. D'ailleurs, l'étape représentée de la manière la plus simplifiée ou approximative constituera le facteur limitant de l'ensemble du modèle, et il sera alors difficile de pouvoir juger de la pertinence des équations plus complexes. De même, le niveau de description du bassin versant et du réseau (pentes, diamètres, longueurs...) doit être en relation avec le niveau de précision du modèle.

(1) HYPOCRAS : boisson faite avec du vin naturel additionné de sucre dans lequel on a fait infuser de la cannelle, du girofle et de la vanille.
Pour toutes ces raisons, nous avons choisi d'établir un modèle conceptuel, utilisant des représentations simples, avec une description globale de l'ensemble du bassin versant et du réseau. Le domaine de validité d'un tel modèle est limité a priori aux petits bassins versants urbains. Nous considérons comme petits et urbains des bassins versants de moins de 100 ha et dont le coefficient d'imperméabilisation est supérieur à 0,2 . Dans ces conditions, il nous paraît acceptable d'avoir une description globale des choses et de pouvoir négliger les débits provenant des surfaces perméables, ainsi que le proposait Desbordes (1974). Mais il est clair que seule l'expérience pourra confirmer ou invalider ces hypothèses.

D'autre part, à partir d'une étude bibliographique préalable (Bertrand-Krajewski 1991a), des choix complémentaires ont été effectués. Nous les présentons ici sommairement, ils seront repris plus en détail dans les paragraphes suivants.

Si nous adoptons des représentations simples pour le transport solide, il est souhaitable, toujours par souci de cohérence interne, d'avoir une complexité comparable pour la partie hydrologique et hydraulique : une approche conceptuelle sera adoptée ici également.

Les particules transportées en suspension au cours d'un événement pluvieux et les particules constituant les dépôts présentent des caractéristiques physiques (diamètre, densité, nature) distinctes. Il faut tenir compte également des particules des eaux usées de temps sec. Nous distinguerons dans HYPOCRAS deux types fondamentaux de particules pour tenter de prendre en compte cette réalité. N'en prendre qu'une conduirait à des simplifications abusives, en prendre davantage introduirait une complexité et une précision fictive qui ne pourraient pas être validées par les données expérimentales disponibles.

Dès lors que nous distinguons deux types de particules, nous sommes amenés à modéliser trois phénomènes qui s'y rattachent et sont interdépendants : suspension, charriage et dépôt. Cela rend immédiatement le modèle plus complexe, et nous en verrons ultérieurement les conséquences pour les calages.

Dans la mesure du possible, nous essayerons d'avoir le plus petit nombre possible de paramètres à caler pour faciliter l'utilisation du modèle. Compte tenu du nombre minimal d'étapes à modéliser, une dizaine de paramètres semble être un minimum en deçà duquel il est difficile d'aller.

La précision attendue des résultats du modèle doit être en relation avec le niveau de description du système et des phénomènes. Des ordres de grandeurs corrects et des évolutions des grandeurs en cohérence avec les observations représentent un objectif minimum. Toutefois, compte tenu des erreurs moyennes atteignant facilement 20 à $30 \%$, voire plus, pour les données d'entrée du modèle, il serait illusoire de chercher une précision supérieure dans les résultats des calculs. Par ailleurs, l'erreur sur les mesures des concentrations servant aux calages peut atteindre aisément 20 à $30 \%$ également, voir $50 \%$, ce qui relativise les conclusions que l'on peut en tirer.

Le modèle HYPOCRAS complet comporte deux parties distinctes (Bertrand-Krajewski 1992b) : 
- une partie temps sec, qui modélise les pollutogrammes des solides en suspension sur des périodes de $24 \mathrm{~h}$ et les variations des dépôts en temps sec;

- une partie temps de pluie qui décrit les phénomènes relatifs à un événement pluvieux.

Dans le cadre de cet article, nous nous limiterons à la partie temps de pluie qui est la plus importante.

\section{Description du modèle}

La partie temps de pluie est construite autour de deux modules (voir fig. 1):

- un module hydrologique et hydraulique pour simuler la transformation pluie-débit à l'exutoire ;

- un module de production et de transfert des solides qui modélise l'ensemble des processus à prendre en compte : accumulation à la surface du bassin versant, entraînement par la pluie, traversée des avaloirs, érosion et sédimentation des dépôts, propagation dans le réseau.

\subsection{Module hydrologique et hydraulique}

\subsubsection{Pluie brute}

Il s'agit du hyétogramme de la pluie, décrit par des intensités en $\mathrm{mm} / \mathrm{h}$ sur des pas de temps de 1 ou $2 \mathrm{~min}$. A l'échelle à laquelle nous travaillons, nous ne prenons en compte ni abattement spatial ni déplacement de la pluie, et nous supposons que la pluie est répartie de manière homogène sur l'ensemble du bassin versant. Cette hypothèse, utilisée dans un souci de simplification, n'est pas toujours vérifiée dans les faits, même pour de petits bassins versants (Schilling 1983, Niemczynowicz 1988).

\subsubsection{Pertes}

Avant ruissellement la pluie subit des pertes : interception par les végétaux, évaporation, infiltration et stockage dans les dépressions du sol. Dans HYPOCRAS, seule la pluie tombant sur les surfaces imperméables est prise en compte (Desbordes 1974, Thibault 1981).

Plusieurs modèles de pertes ont été proposés dans la littérature. Nous retiendrons les deux suivants, en raison de leur simplicité et de leur relativement bonne représentativité pour les surfaces imperméables (Jovanović 1986) :

- une perte initiale PI en $\mathrm{mm}$ et une perte continue constante PCC en $\mathrm{mm} / \mathrm{h}$;

- une perte initiale PI en $\mathrm{mm}$ et une perte continue proportionnelle PCP en $\mathrm{mm} / \mathrm{mm}$ de pluie.

Le programme offre les deux options: selon les cas, l'une ou l'autre se montre la plus satisfaisante pour ajuster les résultats.

\subsubsection{Pluie nette}

La pluie nette correspond à la quantité d'eau qui va effectivement ruisseler sur le bassin versant et se propager

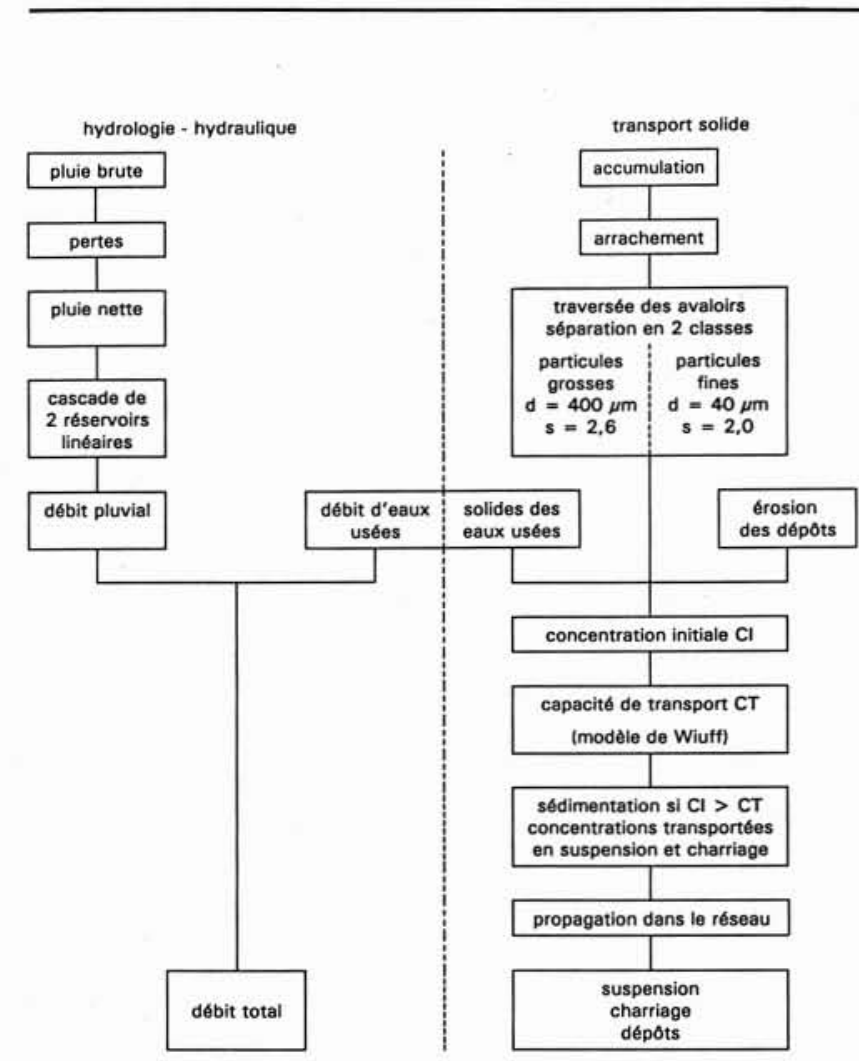

1. Schéma général du modèle HYPOCRAS.

à travers le réseau. Elle est calculée en soustrayant les pertes à la pluie brute :

$$
\begin{array}{rlrl}
\text { Si } \sum_{i=1}^{N} \operatorname{Pb}(i) \leqslant \mathrm{PI} & \operatorname{Pn}(i) & =0 \\
\text { Si } \quad \sum_{i=1}^{N} \mathrm{~Pb}(i)>\mathrm{PI} & \mathrm{Pn}(i) & =\mathrm{Pb}(i)-\mathrm{PCC} \\
\text { ou } & \mathrm{Pn}(i)=\mathrm{Pb}(i) * \mathrm{PCP}
\end{array}
$$

avec

Pn pluie nette $(\mathrm{mm} / \mathrm{h})$

$\mathrm{Pb}$ pluie brute $(\mathrm{mm} / \mathrm{h})$

$\mathrm{N}$ nombre de pas de temps de la pluie.

La pluie nette doit se retrouver intégralement à l'exutoire du réseau (sauf cas particuliers de fuites ou de déversements).

\subsubsection{Ruissellement et propagation dans le réseau}

Ces deux phénomènes sont simulés en une seule étape. En effet, pour de petits bassins versants où les longueurs des collecteurs sont faibles, nous faisons l'hypothèse que l'ensemble bassin versant + réseau peut être décrit de manière globale (Marr 1976, Jovanović 1986). Plusieurs essais nous ont conduit à utiliser une cascade de deux 


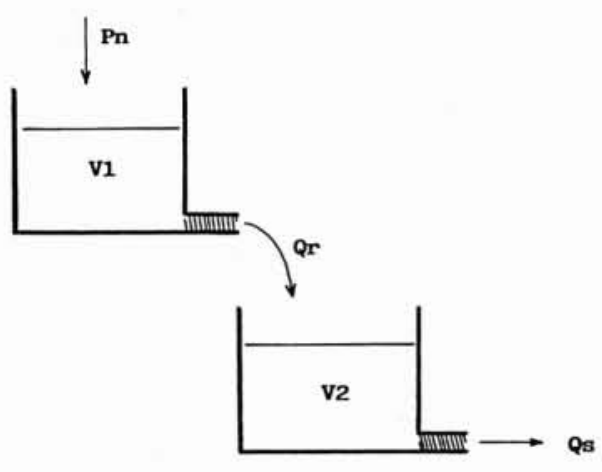

2. Transformation pluie-débit à l'exutoire.

réservoirs linéaires pour représenter au mieux ces phénomènes (fig. 2).

Rappelons que le modèle du réservoir linéaire est établi à partir des deux équations suivantes (pour le premier réservoir) :

— une équation de continuité :

$$
\frac{\mathrm{dV} 1(\mathrm{t})}{\mathrm{dt}}=\operatorname{Pn}(\mathrm{t}) \cdot \mathrm{A} \cdot \mathrm{IMP} \cdot 10^{-1}-\mathrm{Qr}(\mathrm{t})
$$

— une équation de stockage :

$$
\mathrm{V} 1(\mathrm{t})=\mathrm{K} 1 \cdot \operatorname{Qr}(\mathrm{t})
$$

avec

V1 volume stocké à l'instant $\mathrm{t}$ dans le réservoir $1\left(\mathrm{~m}^{3}\right)$

$\mathrm{Pn}$ pluie nette $(\mathrm{mm} / \mathrm{h})$

A superficie du bassin versant (ha)

IMP coefficient d'imperméabilisation du bassin versant

Qr débit de sortie du réservoir $1\left(\mathrm{~m}^{3} / \mathrm{h}\right)$

$\mathrm{t}$ temps (h)

K1 lag-time du réservoir 1 (h).

Après intégration et discrétisation au pas de temps $\Delta \mathrm{t}$, nous obtenons :

$$
\begin{array}{r}
\mathrm{Qr}(n+1 \cdot \Delta \mathrm{t})=\mathrm{C} 12 \cdot \mathrm{Pn}(n+1 \cdot \Delta \mathrm{t}) \cdot \mathrm{A} \cdot \mathrm{IMP} \times \\
\times 10^{-1}+\mathrm{C} 13 \cdot \mathrm{Qr}(n \cdot \Delta \mathrm{t})
\end{array}
$$

avec

$$
\mathrm{C} 13=\exp (-\Delta \mathrm{t} / \mathrm{K} 1) \text { et } \mathrm{C} 12=1-\mathrm{C} 13 .
$$

Le débit d'eaux pluviales Qs à l'exutoire est donné par la relation suivante :

$\mathrm{Qs}(n+1 \cdot \Delta \mathrm{t})=\mathrm{C} 22 \cdot \mathrm{Qr}(n+1 \cdot \Delta \mathrm{t})+\mathrm{C} 23 \cdot \mathrm{Qs}(n \cdot \Delta \mathrm{t})$

avec

$$
\mathrm{C} 23=\exp (-\Delta \mathrm{t} / \mathrm{K} 2) \text { et } \mathrm{C} 22=1-\mathrm{C} 23 .
$$

Le calage des paramètres $\mathrm{K} 1$ et $\mathrm{K} 2$ peut être fait soit par l'utilisateur, soit par une procédure automatique de minimisation d'une fonction objectif. La précision des ajustements, pour les événements étudiés, n'est limitée que par la précision des valeurs de la pluie brute et du modèle de pertes. Un réservoir unique a conduit à des résultats non satisfaisants; l'introduction de réservoirs supplémentaires selon le modèle de Nash par exemple n'a pas apporté d'amélioration significative des résultats. C'est la raison pour laquelle, au vu des simulations réalisées jusqu'à présent, nous avons retenu un modèle à deux réservoirs.

\subsubsection{Hydrogramme à l'exutoire}

Le débit total à l'exutoire est calculé en sommant le débit d'eaux pluviales et le débit d'eaux usées :

$$
\operatorname{Qex}(n \cdot \Delta \mathrm{t})=\operatorname{Qs}(n \cdot \Delta \mathrm{t})+\operatorname{QTS}(n \cdot \Delta \mathrm{t})
$$

avec

Qex débit à l'exutoire $\left(\mathrm{m}^{3} / \mathrm{h}\right)$ à l'instant $\mathrm{t}$

QTS débit d'eaux usées en temps sec $\left(\mathrm{m}^{3} / \mathrm{h}\right)$ durant l'heure h coïncidant avec le pas de temps $n$. $\Delta \mathrm{t}$.

Les débits d'eaux usées en temps sec sont des débits moyens horaires mesurés in situ. En l'absence de mesures, des débits horaires moyens sont calculés à partir du nombre d'habitants raccordés, du volume moyen journalier par habitant et d'une courbe théorique bimodale des débits horaires sur $24 \mathrm{~h}$. Un débit d'infiltration ou de fuite QI peut être introduit.

\subsubsection{Paramètres de calage}

La partie hydrologique et hydraulique comporte donc 4 paramètres :

- la perte initiale PI

- la perte continue PCC ou PCP

- les lag-times des réservoirs $\mathrm{K} 1$ et $\mathrm{K} 2$.

Pour des raisons de commodité des calculs, actuellement seuls les paramètres $\mathrm{K} 1$ et $\mathrm{K} 2$ peuvent être calés automatiquement par le modèle. Les paramètres des pertes sont fixés par l'utilisateur.

\subsection{Partie transport solide}

\subsubsection{Accumulation des solides}

L'accumulation des solides à la surface du bassin versant est représentée par l'équation classique utilisée dans le SWMM (Alley et Smith 1981) :

$$
\frac{\mathrm{dMa}(\mathrm{t})}{\mathrm{dt}}=\mathrm{ACCU} \cdot \mathrm{A} \cdot \mathrm{IMP}-\mathrm{DISP} \cdot \mathrm{Ma}(\mathrm{t})
$$

avec

Ma masse de solides accumulée sur le bassin versant ( $\mathrm{kg})$

ACCU taux d'accumulation (kg.ha- $\left.{ }^{-1} \cdot \mathrm{j}^{-1}\right)$

DISP coefficient de disparition $\left(\mathrm{j}^{-1}\right)$

t temps écoulé depuis la pluie précédente (j).

Lors de la pluie précédente, tous les solides accumulés en surface n'ont pas nécessairement été entraînés vers le réseau. Il peut en subsister une masse résiduelle notée 
MR. Dans ces conditions, et après intégration, l'équation (7) devient :

$$
\begin{aligned}
& \mathrm{Ma}(\mathrm{DTS})= \\
& =\frac{\mathrm{ACCU}}{\mathrm{DISP}} \cdot \mathrm{A} \cdot \mathrm{IMP} \cdot\left(1-\exp \left(\frac{-\mathrm{DISP} \cdot \mathrm{DTS}}{24}\right)\right) \\
& \quad+\mathrm{MR} \cdot\left(\exp \left(\frac{- \text { DISP.DTS }}{24}\right)\right)
\end{aligned}
$$

avec DTS durée de temps sec antérieure à la pluie (h).

Nous voyons immédiatement que la masse Ma tend vers une valeur limite $\mathrm{ML}=\mathrm{ACCU} . \mathrm{A} . \mathrm{IMP} / \mathrm{DISP}$. ACCU représente la production de solides due à l'érosion des chaussées, des pneumatiques, aux retombées atmosphériques, aux déchets divers, etc. DISP correspond à la disparition partielle des solides en raison du vent, du trafic automobile, de la biodégradation, du lavage des voiries, etc. Ces deux paramètres doivent être calés expérimentalement.

\subsubsection{Entraînement par la pluie}

En admettant que l'entraînement des particules par la pluie est proportionnel à la masse disponible et à l'intensité de la pluie, nous pouvons écrire l'équation suivante :

$$
\frac{\mathrm{dMa}(\mathrm{t})}{\mathrm{dt}}=-\mathrm{ARRA} \cdot \mathrm{Ma}(\mathrm{t}) \cdot \operatorname{Ip}(\mathrm{t})
$$

avec

ARRA coefficient d'arrachement $\left(\mathrm{mm}^{-1}\right)$

Ip intensité de la pluie $(\mathrm{mm} / \mathrm{h})$

t temps écoulé depuis le début de la pluie (h).

Cette équation a été utilisée initialement dans le SWMM (Jewell et Adrian 1978, Alley 1981).

Toute particule arrachée par l'impact des gouttes de pluie n'est entraînée vers le réseau que si le ruissellement est suffisamment important. Or le ruissellement est lié à la pluie nette. Comme seule la masse de particules effectivement entrainée vers le réseau nous intéresse, nous prendrons comme intensité effective l'intensité de la pluie nette Pn. En notant MAI la masse accumulée au début de la pluie et en considérant l'intensité de la pluie constante sur l'intervalle de temps $[0, t]$, l'intégration de l'équation (9) conduit à :

$$
M a(t)=M A I \cdot(\exp (- \text { ARRA } \cdot P n \cdot t)) .
$$

En discrétisant cette relation au pas de temps $\Delta t$, la masse entraînée vers le réseau s'écrit :

$$
\begin{aligned}
& \operatorname{MENT}(n+1 \cdot \Delta \mathrm{t})=\operatorname{Ma}(n \cdot \Delta \mathrm{t}) \times \\
& \quad \times\left[1-\exp \left(-\operatorname{ARRA} \cdot \operatorname{Pn}(n+1 \cdot \Delta \mathrm{t}) \cdot \frac{\Delta \mathrm{t}}{60}\right)\right]
\end{aligned}
$$

avec $\Delta t$ en minutes.

A la fin de l'événement pluvieux, la masse résiduelle MR s'écrit :

$\mathrm{MR}=\mathrm{MAI} \cdot\left[\exp \left(-\mathrm{ARRA} \cdot \sum_{i=1}^{N} \operatorname{Pn}\left(\frac{j \cdot \Delta \mathrm{t}}{60}\right)\right)\right]$

avec $\mathrm{N}$ nombre de pas de temps de la pluie.

\subsubsection{Séparation en deux classes de particules}

Au niveau des avaloirs, avant que les solides n'entrent dans le réseau proprement dit, nous distinguons deux classes de particules, dont les caractéristiques ont été fixées d'après les diverses études expérimentales disponibles (Bertrand-Krajewski 1992b). Les deux classes utilisées par HYPOCRAS ont été établies pour représenter :

- les particules fines (type $1: d_{50}=40 \mu \mathrm{m}$, densité 2,0) transportées majoritairement en suspension ;

- les particules plus grosses (type 2: $d_{50}=400 \mu \mathrm{m}$, densité 2,6) qui représentent les dépôts et qui sont transportées par charriage ou en suspension selon les conditions hydrauliques.

En première approximation, les particules des eaux usées seront assimilées aux particules fines des eaux pluviales, par souci de simplification des calculs. En fait, les particules fines des eaux usées ont une densité de 1,1 à 1,8 et les particules fines des eaux pluviales une densité moyenne de 2,4 à 2,6 (Chebbo et al. 1990) : nous avons choisi une densité moyenne de 2,0.

Ce choix de deux classes est naturellement réducteur de la réalité, mais il offre plusieurs avantages:

- grâce aux deux types de solides considérés comme représentatifs, HYPOCRAS peut simuler les deux réalités physiques différentes que sont les dépôts et la suspension ;

- en nous limitant à deux types de solides, le calage et la validation expérimentale restent possibles avec les données expérimentales disponibles, ce qui ne serait pas le cas si nous avions utilisé une description granulométrique plus complète avec plusieurs classes. Les calculs sont également plus courts avec deux classes.

Au vu des résultats obtenus, cette approche nous paraît suffisante en l'état actuel.

A la sortie des avaloirs, nous pouvons écrire, en notant en indice $i$ le type de particules:

$$
\operatorname{MENT}_{i}(n \cdot \Delta \mathrm{t})=\text { Fpent }_{i} \cdot \operatorname{MENT}(n \cdot \Delta \mathrm{t})
$$

avec Fpent ${ }_{i}$ la fraction en poids des particules de type i entraînées vers le réseau.

D'après les mesures expérimentales disponibles, nous admettrons, en première approximation, que :

$$
\mathrm{Fpent}_{1}=\mathrm{Fpent}_{2}=0,5 \text {. }
$$

\subsubsection{Erosion des dépôts antérieurs}

La modélisation utilisée pour le temps sec est reprise pour le temps de pluie: l'érosion des dépôts est supposée proportionnelle à la masse déposée et au débit. En admettant que les dépôts contiennent des particules des deux types, nous avons :

$$
\frac{\mathrm{dMD}_{i}(\mathrm{t})}{\mathrm{dt}}=-\mathrm{Kero}_{i} \cdot \mathrm{Qex}(\mathrm{t}) \cdot \mathrm{MD}_{i}(\mathrm{t})
$$

avec

$\mathrm{MD}_{i}$ masse érodable de dépôts de type $\mathrm{i}$ en début de pluie $(\mathrm{kg})$

Kero $_{i}$ coefficient d'érosion des dépôts $\left(\mathrm{m}^{-3}\right)$

$\mathrm{t}$ temps (h). 


\section{J.L. BERTRAND-KRAJEWSKI}

Après intégration et discrétisation, nous pouvons déterminer la masse érodée :

$$
\begin{aligned}
& \operatorname{MERO}_{\mathrm{i}}(n+1 \cdot \Delta \mathrm{t})= \\
& \quad=\mathrm{MD}_{\mathrm{i}} \cdot\left[1-\exp \left(-\mathrm{Kero}_{\mathrm{i}} \cdot \operatorname{Qex}(n+1 \cdot \Delta \mathrm{t}) \cdot \frac{\Delta \mathrm{t}}{60}\right)\right]
\end{aligned}
$$

avec $\mathrm{MERO}_{i}$ masse érodée $(\mathrm{kg})$.

\subsubsection{Concentration initiale}

Nous appelons concentration initiale la concentration en solides provenant des eaux de ruissellement, des eaux usées et de l'érosion des dépôts. En temps sec, les valeurs habituellement disponibles sont des valeurs moyennes horaires tant pour les débits que pour les flux. En notant respectivement QTS(h) et FTS(h) le débit et le flux moyens de temps sec durant l'heure h coïncidant avec le pas de temps $n \cdot \Delta t$, nous avons :

$\mathrm{CENT}_{1}(n \cdot \Delta \mathrm{t})=$

$=\frac{\left(\left[\operatorname{MENT}_{1}(n \cdot \Delta \mathrm{t})+\operatorname{MERO}_{1}(n \cdot \Delta \mathrm{t})\right] \cdot \frac{\Delta \mathrm{t}}{60}\right)+\operatorname{FTS}(\mathrm{h})}{\operatorname{Qex}(n \cdot \Delta \mathrm{t})}$

$\mathrm{CENT}_{2}(n \cdot \Delta \mathrm{t})=$

$$
=\frac{\left[\operatorname{MENT}_{2}(n \cdot \Delta \mathrm{t})+\operatorname{MERO}_{2}(n \cdot \Delta \mathrm{t})\right] \cdot \Delta \mathrm{t}}{60 \cdot \operatorname{Qex}(n \cdot \Delta \mathrm{t})}
$$

$\operatorname{CENT}(n \cdot \Delta \mathrm{t})=\operatorname{CENT}_{1}(n \cdot \Delta \mathrm{t})+\operatorname{CENT}_{2}(n \cdot \Delta \mathrm{t})$

avec

CENT $_{i}$ concentration initiale en particule de type $i$ $\left(\mathrm{kg} / \mathrm{m}^{3}\right)$

CENT concentration initiale totale $\left(\mathrm{kg} / \mathrm{m}^{3}\right)$

FTS flux de temps sec $(\mathrm{kg} / \mathrm{h})$.

Il s'agit à présent de déterminer si une telle concentration est effectivement transportable par le débit Qex.

\subsubsection{Représentation du réseau}

Afin de conserver une représentation simple et globale du réseau, nous proposons de le représenter par un collecteur équivalent unique (voir fig. 3).

Ce collecteur équivalent est défini par deux paramètres seulement :

— un diamètre équivalent Deq, égal à celui du collecteur à l'exutoire du réseau ;

- une pente équivalente Ieq, égale à la pente moyenne des collecteurs ou du bassin versant. La pente joue un rôle important dans le modèle, notamment pour la répartition entre la suspension et le charriage.

Le collecteur équivalent n'a pas de longueur : cela n'a aucune importance pour le fonctionnement du modèle proprement dit ; le calcul du transfert des solides à travers le réseau au moyen d'un réservoir linéaire introduira de manière indirecte une longueur pour le collecteur équivalent.

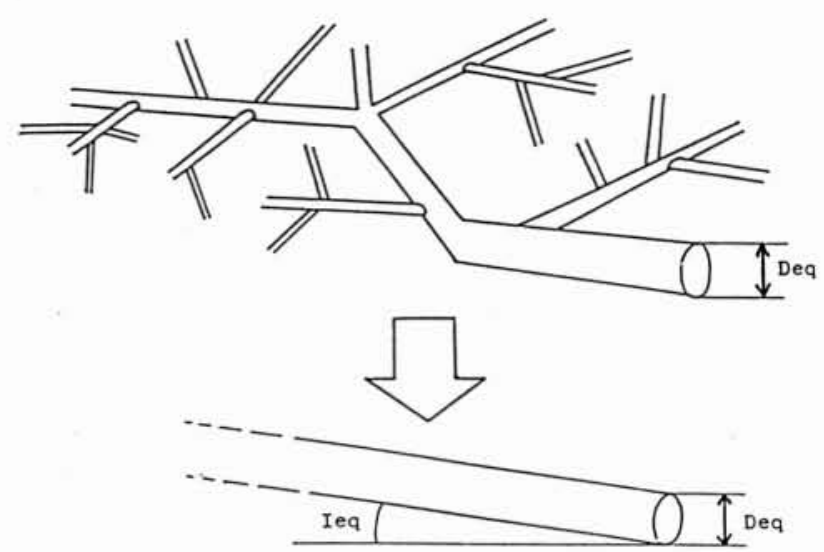

3. Représentation simplifiée du réseau pour le transport solide.

\subsubsection{Capacité de transport}

Le débit Qex à travers le collecteur équivalent possède une capacité de transport $\mathrm{CT}$, exprimée en $\mathrm{kg} / \mathrm{m}^{3}$, audelà de laquelle il y a sédimentation. La plupart des équations de transport solide utilisées en hydrologie urbaine proviennent de I'hydraulique fluviale. Ceci ne va pas sans poser de problèmes en réseau d'assainissement, mais des équations véritablement spécifiques aux réseaux d'assainissement restent encore à trouver. Nous sommes donc contraints de nous orienter vers une équation classique de transport solide. Parmi les très nombreuses équations possibles, nous avons cherché un modèle simple et utilisable sans avoir à introduire de paramètres supplémentaires. Nous avons ainsi retenu le modèle de R. Wiuff (1985), établi à partir de considérations énergétiques, pour les raisons suivantes :

— formulation mathématique simple de la capacité de transport ;

- expressions similaires pour la concentration transportée en suspension et pour la concentration du transport solide total. Par différence, il est possible de déterminer le charriage ;

- résultats très voisins de ceux obtenus par le modèle classique d'Hengelund-Hansen.

Parmi les inconvénients de ce modèle, il faut mentionner :

— son absence de vérification pour les conduites circulaires ;

- son manque de validation pour les distributions granulométriques étendues ;

- sa vérification expérimentale limitée aux particules comprises entre 190 et $930 \mu \mathrm{m}$.

Le modèle initial s'écrit :

$$
\mathrm{CT}=\eta \cdot \frac{\rho \cdot U \cdot I \cdot \rho_{s}}{\left(\rho_{s}-\rho\right) \cdot W}
$$


avec

CT capacité de transport $\left(\mathrm{kg} / \mathrm{m}^{3}\right)$

$\rho$ masse volumique de l'eau $\left(\mathrm{kg} / \mathrm{m}^{3}\right)$

$\rho_{\mathrm{s}} \quad$ masse volumique des particules $\left(\mathrm{kg} / \mathrm{m}^{3}\right)$

$U$ vitesse moyenne de l'écoulement $(\mathrm{m} / \mathrm{s})$

$l$ pente

$W$ vitesse de chute des particules $(\mathrm{m} / \mathrm{s})$

$\eta$ coefficient de rendement.

Pour la suspension et le transport total, nous avons respectivement :

$$
\begin{gathered}
\eta_{s}=0.016 \cdot \frac{\tau}{\left(\rho_{s}-\rho\right) \cdot g \cdot d} \\
\eta_{t}=\eta_{s} \cdot Z \\
Z=\frac{W}{\mathrm{Kar} \cdot U^{*}}
\end{gathered}
$$

avec

avec

$\eta$, çoefficient de rendement de la suspension

$\tau$ contrainte de cisaillement $\left(\mathrm{N} / \mathrm{m}^{2}\right)$

$g$ accélération de la pesanteur $\left(\mathrm{m} / \mathrm{s}^{2}\right)$

$d$ diamètre des particules $(\mathrm{m})$

$\eta$, coefficient de rendement du transport total

Z nombre de Rouse des particules

Kar constante de Von Karman

$U^{*}$ vitesse de frottement $(\mathrm{m} / \mathrm{s})$.

Si $Z$ est supérieur à 1 , il est possible de calculer le charriage avec :

$$
\eta_{i}=\eta_{s} \cdot(Z-1) .
$$

Wiuff n'ayant pas étudié l'adaptation de son modèle aux granulométries étendues (Wiuff 1991), nous avons proposé des modifications pour prendre en compte différentes classes granulométriques, ce qui conduit à la relation suivante :

$$
\mathrm{CT}=\frac{\rho \cdot U \cdot l}{\sum_{i=1}^{N}\left(\frac{F p_{i} \cdot\left(\rho_{\mathrm{si}}-\rho\right) \cdot W_{i}}{\rho_{\mathrm{si}} \cdot \eta_{i}}\right)}
$$

avec

$\rho_{\mathrm{si}} \quad$ masse volumique des particules de classe $i$

$\eta_{1}$ coefficient de rendement de la classe $i$

Fp, fraction en poids de la classe $i$.

CT représente la capacité de transport en suspension ou totale selon que l'on prend $\eta_{s}$ ou $\eta_{t}$.

Connaissant le débit Qex, le diamètre Deq et la pente leq. toutes les valeurs intermédiaires des calculs peuvent être déterminées facilement. Nous en déduisons alors les capacités de transport totale, en suspension et par charriage, respectivement CTtot, CTsusp et CTchar.

Les équations ainsi établies fournissent des résultats cohérents avec ceux d'autres formules de transport solide plus complexes ou fondées sur des approches physiques différentes (Briat 1989).

\subsubsection{Concentration transportée}

Nous disposons, à chaque pas de temps, de deux grandeurs : la concentration initiale CENT et la capacité de transport CTtot. Si CENT $<$ CTtot, toutes les particules sont transportées en fonction des résultats donnés par le modèle de Wiuff modifié. Si CENT $>$ CTtot, il y a sédimentation partielle des particules. Les calculs sont menés par itérations successives de manière à vérifier pour chaque pas de temps le principe de conservation de la masse entre les particules transportées en suspension et en charriage et les particules déposées.

Nous obtenons alors les concentrations transportées totale, en suspension et en charriage, notées respectivement Ctrans, Csusp et Cchar :

$$
\begin{aligned}
& \text { Csusp }=\sum\left(\frac{\mathrm{Fpt}_{i} \cdot \text { Ctrans }}{Z_{i}}\right) \\
& \text { Cchar }=\sum\left(\frac{\mathrm{Fpt}_{i} \cdot \text { Ctrans } \cdot\left(Z_{i}-1\right)}{Z_{i}}\right)
\end{aligned}
$$

avec

$Z_{i} \quad$ nombre de Rouse des particules de la classe $i$

$\mathrm{Fpt}_{\mathrm{i}} \quad$ fraction en poids des particules transportées de la classe $i$.

\subsubsection{Transit des particules}

Une fois déterminées les concentrations en suspension et en charriage, les deux modes de transport sont séparés dans la suite des calculs. Le transfert des solides en suspension et en charriage est simulé par un modèle de type réservoir linéaire. Le collecteur équivalent se voit ainsi attribuer, de manière indirecte, une certaine longueur que les solides parcourent en un temps égal au lagtime.

Dans les conditions habituelles, le flux de temps sec est mesuré directement à l'exutoire du bassin versant. Avant passage à travers le réservoir linéaire, les solides de type 1 correspondant aux eaux usées de temps sec sont soustraits de la suspension totale. En sortie du réservoir, ils sont à nouveau ajoutés à la suspension pour donner la suspension totale à l'exutoire.

L'ensemble de ces phénomènes est représenté par les équations suivantes:

$$
\begin{aligned}
& \operatorname{CsuspE}(n+1 \cdot \Delta \mathrm{t})= \\
& \quad=\operatorname{CsuspE}(n+1 \cdot \Delta \mathrm{t})-\left(\frac{\mathrm{FTS}(\mathrm{h})}{\mathrm{Qex}(n+1 \cdot \Delta \mathrm{t})}\right) \\
& \operatorname{CsuspS}^{\prime}(n+1 \cdot \Delta \mathrm{t})= \\
& =\mathrm{C} 32 \cdot \operatorname{CsuspS}^{\prime}(n \Delta \mathrm{t})+\mathrm{C} 33 \cdot \operatorname{CsuspE}^{\prime}(n+1 \cdot \Delta \mathrm{t})
\end{aligned}
$$

$\operatorname{CcharS}(n+1 \cdot \Delta \mathrm{t})=$

$$
=\mathrm{C} 42 \cdot \mathrm{CcharS}(n \Delta \mathrm{t})+\mathrm{C} 43 \cdot \mathrm{CcharE}(n+1 \cdot \Delta \mathrm{t})
$$

$\operatorname{CsuspS}(n+1 \cdot \Delta \mathrm{t})=$

$$
=\operatorname{CsuspS}^{\prime}(n+1 \cdot \Delta \mathrm{t})+\left(\frac{\mathrm{FTS}(\mathrm{h})}{\operatorname{Qex}(n+1 \cdot \Delta \mathrm{t})}\right)
$$

avec

CsuspE' concentration en suspension en entrée après soustraction du temps sec 
CsuspE concentration en suspension en entrée avant soustraction du temps sec

CsuspS' concentration en suspension en sortie avant addition du temps sec

CsuspS concentration en suspension en sortie après addition du temps sec

CcharE concentration en charriage en entrée

CcharS concentration en charriage en sortie

$\mathrm{C} 13=\exp (-\Delta \mathrm{t} / \mathrm{K}$ susp $)$ et $\mathrm{C} 12=1-\mathrm{C} 13$

avec Ksusp lag-time de la suspension

$\mathrm{C} 23=\exp (-\Delta \mathrm{t} / \mathrm{Kchar})$ et $\mathrm{C} 22=1-\mathrm{C} 23$

avec Kchar lag-time du charriage .

Dans un premier temps, et bien que cela soit a priori irréaliste compte tenu de la différence entre les modes de transport, nous avons adopté Ksusp $=$ Kchar. Ce choix se justifie par les données dont nous disposions pour caler le modèle. En effet, les prélèvements classiques effectués en réseau ne prennent pas en compte le charriage mais uniquement la suspension : nous ne pourrons donc pas caler directement les valeurs du transport par charriage: Par ailleurs, les quelques données disponibles aujourd'hui sur le charriage concernent davantage les charges transportées au cours d'un événement que l'évolution du charriage au cours du temps. Or le modèle du réservoir linéaire, par définition, est conservatif : quelle que soit la valeur de Kchar, il n'y a pas de modification de la charge totale transportée par charriage. Plutôt que de chercher une valeur impossible à caler pour Kchar, nous préférons donc fixer arbitrairement $\mathrm{Kchar}=\mathrm{Ksusp}$, en attendant que des mesures expérimentales nous permettent de fixer des valeurs plus appropriées.

\subsubsection{Valeurs de sortie}

A l'exutoire du réseau, le modèle fournit les résultats suivants :

- la concentration en suspension en fonction du temps (pollutogramme)

- la charge totale transportée par charriage

- les dépôts à la fin de l'événement pluvieux par type de particules.

En considérant, dans la meilleure des hypothèses, que les mesures expérimentales représentent correctement la suspension (les techniques habituelles de prélèvement et la représentativité des échantillons sont telles que cette hypothèse est loin d'être vérifiée), l'ajustement du modèle se fera sur le pollutogramme de la suspension. Les résultats obtenus en charriage, compte tenu des données disponibles, ne pourront être appréciés que du point de vue de leur ordre de grandeur. Il en va de même pour les dépôts. Nous touchons là directement aux limites rencontrées par tous les modèles de qualité en hydrologie urbaine : le manque de données pour caler l'ensemble des phénomènes à prendre en considération.

\subsubsection{Paramètres du modèle}

La partie transport solide comporte 10 paramètres, que l'on peut regrouper en trois familles:

$1:$ les paramètres liés au réseau proprement dit : - les coefficients d'érosion $\mathrm{Kero}_{1}$ et $\mathrm{Kero}_{2}\left(\mathrm{~m}^{-3}\right)$
- la pente équivalente Ieq

- le lag-time Ksusp ( $\mathrm{min}$ )

$2:$ les paramètres liés au bassin versant :

- le taux d'accumulation ACCU ( $\mathrm{kg} / \mathrm{j} / \mathrm{ha})$

- le coefficient de disparition DISP $\left(\mathrm{j}^{-1}\right)$

- le coefficient d'arrachement ARRA $\left(\mathrm{mm}^{-1}\right)$

$3:$ les paramètres représentant les conditions initiales :

- la masse résiduelle MR $(\mathrm{kg})$

- les masses déposées initiales $\mathrm{MD}_{1}$ et $\mathrm{MD}_{2}(\mathrm{~kg})$.

Les grandeurs suivantes sont considérées comme étant des grandeurs connues et ne font pas l'objet de calages :

- la surface du bassin versant A (ha)

- le coefficient d'imperméabilisation IMP

- la durée de temps sec antérieure DTS (h)

- les diamètres et densités des particules $d_{1}, d_{2}(\mu \mathrm{m})$,

$s_{1}$ et $s_{2}$

- les fractions en poids des particules entraînées Fpent ${ }_{1}$ et Fpent 2

- le coefficient de Manning-Strickler du collecteur équivalent $\mathrm{Kms}=70$.

\section{Calage et validation}

Le modèle HYPOCRAS a été calé sur trois bassins versants aux caractéristiques différentes rassemblées dans le tableau 1 . Tous sont équipés de réseaux unitaires, et présentent un habitat de type résidentiel peu dense.

\subsection{Réseau de Mantes-la-Ville}

Le réseau et la campagne expérimentale ont fait l'objet d'un rapport d'étude du Laboratoire Régional de l'Ouest Parisien (Laveuf et al. 1980). Après critique, validation et préparation des données (Bertrand-Krajewski 1991b), 6 événements pluvieux ont pu être retenus et simulés avec HYPOCRAS. Les événements $n^{\circ} 039$ et 061 sont reproduits figure 4 et figure 5. Dans les deux cas, les hydrogrammes sont correctement simulés. Le pollutogramme 039 est satisfaisant, et a servi comme événement de référence pour le calage des paramètres. Le pollutogramme 061 présente des fluctuations assez importantes des concentrations calculées, ce que les données expérimentales, obtenues par échantillons proportionnels au débit, ne permettent pas de retrouver. Il y a néanmoins un bon accord entre les charges mesurées et calculées, comme indiqué dans le tableau 2.

Les valeurs du charriage sont faibles, mais cohérentes avec les observations. En effet, Laveuf et al. (1980) indiquent que le réseau de Mantes-la-Ville, en raison de ses fortes pentes toujours supérieures à $1 \%$, ne contient pas de dépôts. Les simulations réalisées avec HYPOCRAS permettent de retrouver ces observations :

- les valeurs de $\mathrm{MD}_{1}$ et $\mathrm{MD}_{2}$ sont toujours nulles

- il n'y a pas de formation de dépôts, quel que soit l'épisode pluvieux simulé

- en raison des pentes importantes, quasiment tous les solides peuvent être transportés en suspension durant les pluies, le charriage restant très faible. 
Tableau 1. - Caractéristiques des bassins versants étudiés.

\begin{tabular}{|l|c|c|c|}
\hline \multicolumn{1}{|c|}{ Bassin } & Mantes-la-Ville & Entzheim & Dundee \\
\hline Superficie (ha) & 72 & 40 & 81 \\
\hline Coeff. imperméabilisation & 0,39 & 0,39 & 0,40 \\
\hline Pente moyenne & 0,0318 & 0,002 & 0,025 \\
\hline Nombre d'habitants & 4800 & 1650 & 2500 \\
\hline
\end{tabular}

Tableau 2.

Charges en solides transportés

à Mantes-la-Ville.

\begin{tabular}{|c|c|c|}
\hline Événement pluvieux & 039 & 061 \\
\hline Charge en suspension calculée $(\mathrm{kg})$ & 66,3 & 145,5 \\
\hline Charge en suspension mesurée $(\mathrm{kg})$ & 63,5 & 155,5 \\
\hline Charge en charriage calculée $(\mathrm{kg})$ & 0,9 & 2,6 \\
\hline
\end{tabular}

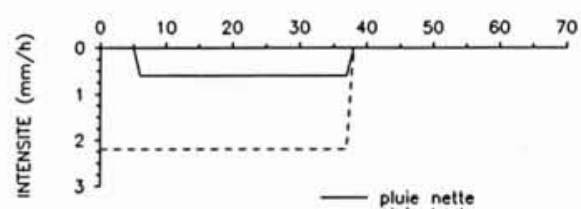

$\overline{\text { p.. pluie nette }}$ pluie brute
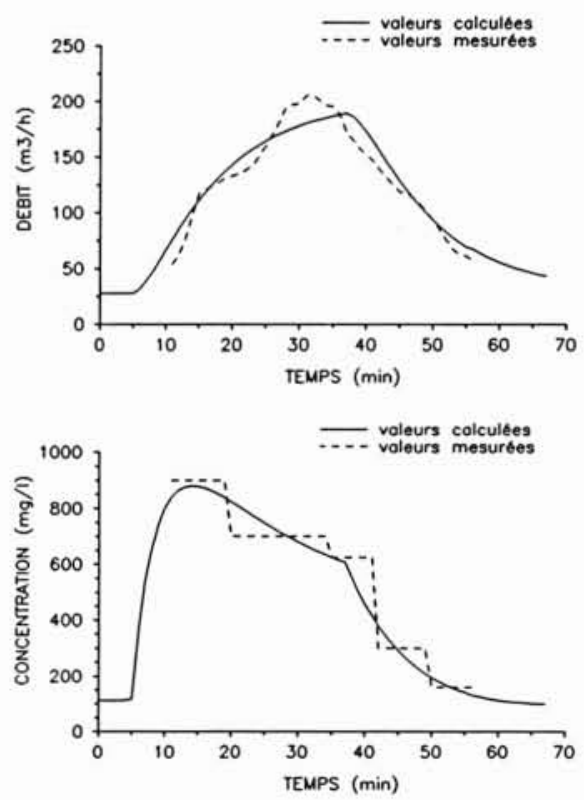

4. Simulation de l'événement 039 (Mantes-la-Ville).

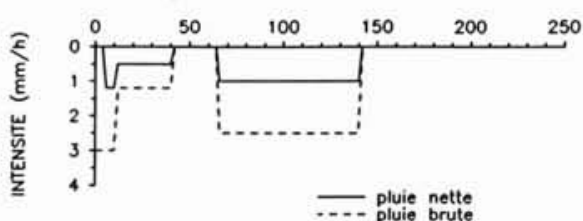

-..- pluie nette
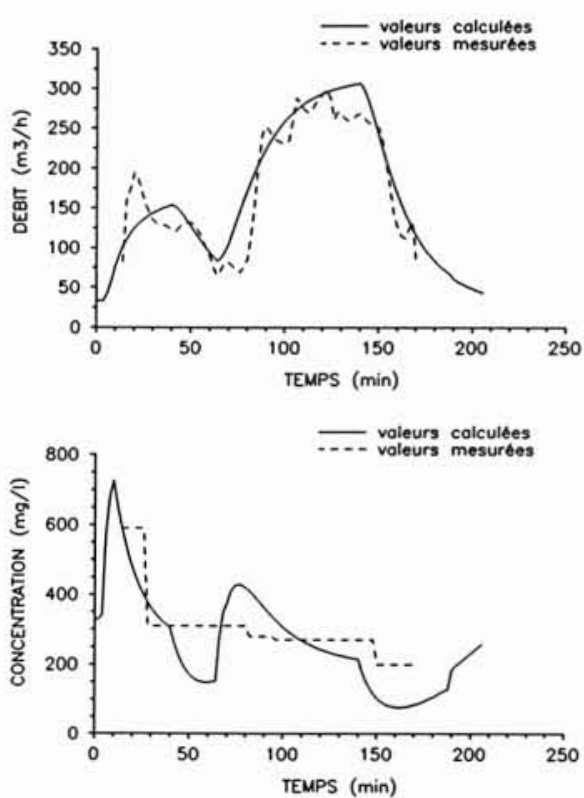

5. Simulation de l'événement 061 (Mantes-la-Ville). 


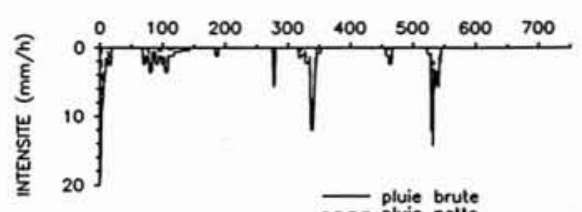

-... pluie brute
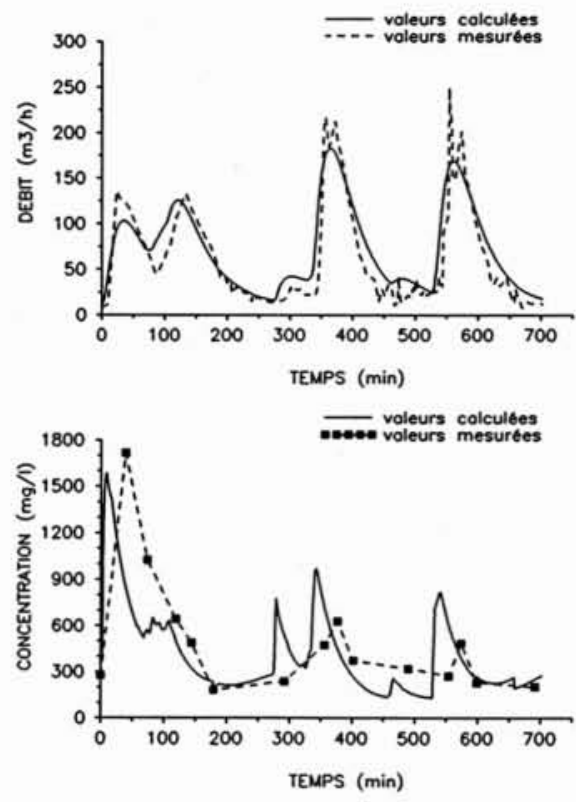

6. Simulation de l'événement 022 (Entzheim).
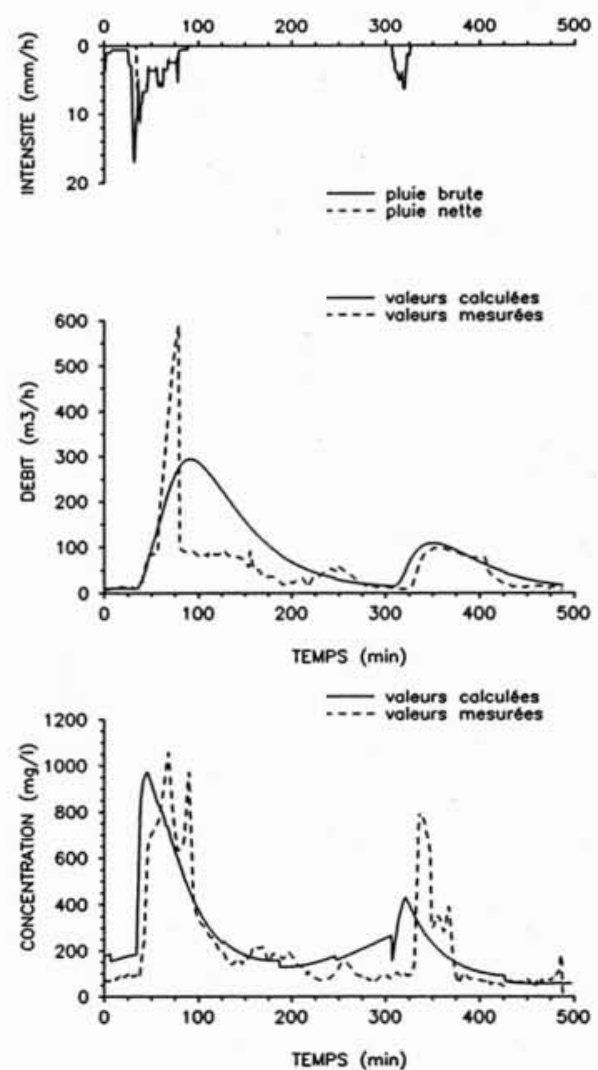

7. Simulation de l'événement 1301 (Entzheim).
Ces premiers résultats, obtenus dans le cas d'un réseau sans dépôts, ont permis de valider les approches mises en œuvre dans HYPOCRAS et d'envisager des calages sur d'autres sites.

\subsection{Réseau d'Entzheim}

Plusieurs campagnes de mesure ont été réalisées sur le site d'Entzheim entre 1986 et 1988 par l'Ecole Nationale des Ingénieurs des Travaux Ruraux et des Techniques Sanitaires de Strasbourg (Bellefeur 1989). A partir des enregistrements d'origine des données expérimentales, nous avons pu sélectionner les épisodes pluvieux les plus intéressants et les mettre sous une forme utilisable par HYPOCRAS (Bertrand-Krajewski et Bellefleur 1991). Huit événements ont été simulés : les événements n 022 et $130 \_1$ sont reproduits figure 6 et figure 7.

L'épisode pluvieux 022 est particulièrement intéressant, car il présente une durée importante (plus de $9 \mathrm{~h}$ ), avec différents pics de pluie. L'hydrogramme est reproduit de manière tout à fait satisfaisante. Sur le pollutogramme calculé, les pics de concentration successifs et décroissants sont correctement simulés, avec une sensibilité marquée vis-à-vis des pointes d'intensité de la pluie. Une pluie aussi complexe permet de tester les capacités du modèle, et les résultats obtenus ici sont encourageants. La charge transportée en charriage est faible $(1,1 \mathrm{~kg})$, mais s'explique par une sédimentation importante des solides de type 2: $65 \mathrm{~kg}$ de grosses particules se sont déposés, tandis que $36 \mathrm{~kg}$ de particules fines, provenant des dépôts de temps sec, ont été érodés. Il en résulte un flux de solides à l'exutoire composé majoritairement de particules fines, ce qui corrobore les observations.

Le pollutogramme mesuré de l'événement 130_1 a été obtenu avec un turbidimètre dont les valeurs ont été corrélées avec les concentrations en solide en suspension des échantillons obtenus avec un préleveur automatique. Cette corrélation a été établie sur le premier pic uniquement. On constate que le pollutogramme calculé est relativement bien ajusté, sauf pour le deuxième pic de concentration. Sur ce second pic, l'échantillon du préleveur avait une concentration de $100 \mathrm{mg} / \mathrm{l}$ environ et le turbidimètre indiquait une valeur correspondant à une concentration de $800 \mathrm{mg} / \mathrm{l}$. Quant au modèle, la concentration calculée est de $400 \mathrm{mg} / \mathrm{l}$. Cet exemple montre toute la difficulté à caler correctement un modèle alors que les données expérimentales sont très dispersées et d'une précision toute relative. Plus que sur les concentrations ponctuelles, le calage doit se faire sur l'allure générale des pollutogrammes et sur les ordres de grandeur des concentrations calculées.

D'autre part, on notera qu'en raison d'une erreur de mesure des débits sur le premier pic, l'hydrogramme calculé a été calé sur le deuxième pic.

Sur l'ensemble des événements simulés sur le site d'Entzheim, nous pouvons constater que

- les pollutogrammes de la suspension sont correctement reproduits

- la forte proportion de particules fines de type 1 à l'exutoire est simulée 
- l'augmentation du dépôt constitué de grosses particules de type 2 est mis en évidence lors de chacune des pluies étudiées. Ceci corrobore les observations faites sur le réseau. où les dépôts annuels sont évalués à 15 tonnes environ.

\subsection{Réseau de Dundee}

Le bassin versant Upper Perth Road à Dundee (Ecosse) a fait l'objet d'études et de mesures importantes concernant les solides transportés et les dépôts menées par le Dundee Institute of Technology (Ashley et al. 1989, 1990). Nous avons pu disposer d'une partie des mesures réalisées, et huit événements pluvieux ont été sélectionnés (BertrandKrajewski 1992a). Les événements n 003 et 007 sont reproduits figure 8 et figure 9.

En considérant l'ensemble des épisodes pluvieux simulés, les valeurs calculées sont satisfaisantes tant pour les hydrogrammes que pour les pollutogrammes. Contrairement à Entzheim, on n'observe pas ici de tendance nette d'une augmentation des dépôts de type 2 : leur masse reste globalement stable. Cette constatation est à rapprocher des observations in situ, qui, sur une période de plusieurs mois, $\mathrm{n}$ 'ont mis en évidence ni augmentation ni diminution significative des dépôts dans le réseau. Ceci se traduit aussi par des charges transportées par charriage légèrement plus élevées qu'à Entzheim. Cependant, compte tenu des pentes des collecteurs, une part plus importante des grosses particules est transportée par charriage.

\subsection{Comportement du modèle et limites des calages}

Une première analyse de sensibilité a permis de mieux comprendre l'influence des divers paramètres sur le fonctionnement du modèle et les résultats des calculs. Ainsi, les trois familles de paramètres indiquées au paragraphe 3.2.11 jouent un rôle particulier. En exploitant l'ensemble des simulations réalisées sur les différents bassins, on trouve, par ordre croissant de sensibilité sur les résultats :

- les paramètres relatifs au réseau proprement dit

- les paramètres relatifs au bassin versant

- les paramètres de conditions initiales.

Il est possible d'en déduire quelques pistes pour des calages ultérieurs et pour un emploi opérationnel du logiciel :

- en l'état actuel du modèle et des connaissances, un calage site par site reste indispensable. II faut donc pouvoir disposer de mesures expérimentales.

- les paramètres liés au réseau et au bassin versant peuvent être calés assez rapidement en se fondant

a) sur des valeurs moyennes trouvées dans la littérature b) sur une série de 5 ou 6 événements pluvieux correctement enregistrés.

Pour disposer d'une telle série d'événements, l'expérience montre qu'il est nécessaire de conduire une campagne de mesure expérimentale sur une année minimum, voire deux.
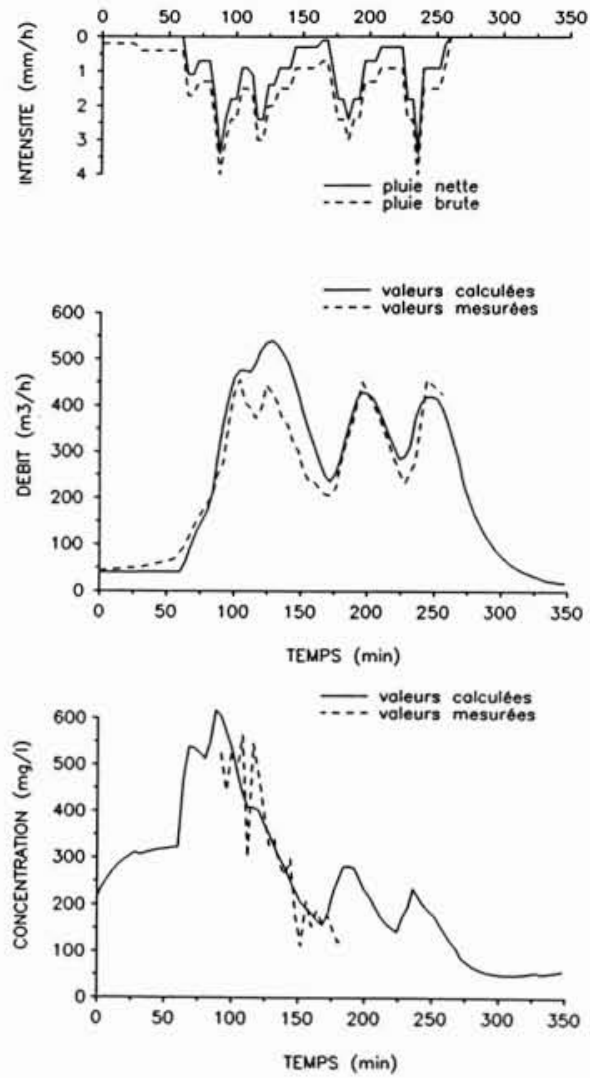

8. Simulation de lévénement 003 (Dundee).
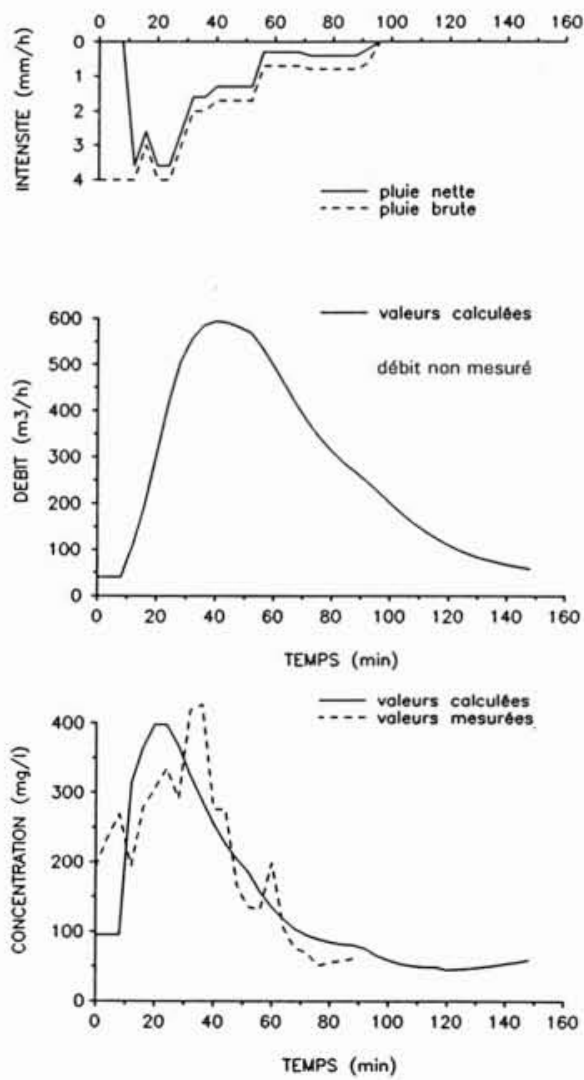

9. Simulation de l'événement 007 (Dundee). 
A moins que des progrès significatifs sur la fiabilité des mesures ne soient réalisés :

- les paramètres représentant les conditions initiales jouent un rôle essentiel dans la détermination des charges rejetées. Or leur évaluation reste très délicate et incertaine : évaluer la masse de solide résiduelle MR sur le bassin versant après un épisode pluvieux est un problème extrêmement difficile, et les techniques de mesure font cruellement défaut; quant aux masses $\mathrm{MD}_{i}$ de dépôts érodables ou mobilisables dans le réseau, si des progrès ont été réalisés en métrologie, des difficultés importantes subsistent :

a) la mesure du dépôt total ne permet pas toujours de séparer les dépôts de type 1 des dépôts de type 2 ;

b) la mesure du dépôt total ne permet pas de connaître la fraction mobilisable ou érodable pour un événement pluvieux donné. Or cette fraction mobilisable peut être extrêmement faible (quelques centaines de kilogrammes à Entzheim, à comparer aux quelques tonnes de dépôts présents dans les collecteurs).

\section{Conclusion}

Le modèle HYPOCRAS est un modèle simple qui cherche à simuler les phénomènes principaux du transport solide en réseau d'assainissement unitaire pour de petits bassins versants urbains. Il fait une distinction entre les particules fines $\left(d_{50}=40 \mu \mathrm{m}\right)$ transportées essentiellement en suspension, et les particules plus grosses $\left(\mathrm{d}_{50}=400 \mu \mathrm{m}\right)$ qui constituent des dépôts plus ou moins permanents et qui sont transportées essentiellement par charriage.

Concernant les pollutogrammes et les charges en suspension, le modèle donne des résultats raisonnablement satisfaisants, compte tenu des incertitudes des mesures de terrain et des approximations utilisées dans les équations. Les principaux phénomènes (accumulation, entraînement par la pluie, érosion des dépôts et transport) sont reproduits de façon réaliste et les ordres de grandeur obtenus sont satisfaisants. L'augmentation du dépôt constitué de grosses particules semble correcte également, mais il est plus difficile de vérifier expérimentalement ce point particulier du modèle. La même remarque est valable pour le charriage.

Cette première version d'HYPOCRAS est certainement perfectible, et demande bien évidemment des calages et des vérifications complémentaires sur des données expérimentales plus nombreuses.

Ces vérifications ultérieures devraient être riches d'enseignement en confortant, nous l'espérons, certains aspects du modèle et en suggérant des améliorations, voire des remises en cause, de certains des concepts mis en œuvre. En effet, ce n'est qu'à travers des confrontations successives entre modélisation et mesures de terrain qu'un perfectionnement et une meilleure représentativité du modèle seront réalisés, et que nous pourrons passer d'un modèle cognitif à un modèle prévisionnel opérationnel.

Comme beaucoup d'autres modèles de qualité en réseau d'assainissement, HYPOCRAS est confronté à une double difficulté :
- son calage nécessite la connaissance de valeurs dont certaines, qui jouent un rôle essentiel dans les calculs, sont très difficilement accessibles à la mesure. C'est le cas notamment de la masse résiduelle en surface et de la masse des dépôts dans les collecteurs ;

- la prise en compte des principaux phénomènes physiques impliqués dans le transport solide conduit à modéliser toute une succession d'étapes de calcul, depuis l'accumulation en surface jusqu'au rejet à l'exutoire. A chaque étape, un ou plusieurs paramètres sont introduits. Or, en l'état actuel, les seules grandeurs facilement accessibles à la mesure sont la pluie, le débit et les solides en suspension. Avec ces trois grandeurs, il nous faut caler une dizaine de paramètres. Il y a là une difficulté majeure, qui ne pourra être progressivement résolue que par le développement des mesures de terrain.

Il nous semble ainsi indispensable de mener un certain nombre d'expérimentations pour progresser dans la connaissance du transport solide :

- mesures à l'entrée des avaloirs, pour mieux quantifier les masses et les caractéristiques physiques des particules entraînées avec le ruissellement. Il sera possible alors de préciser tout à la fois les diamètres et densités des particules entrant réellement dans les avaloirs, et les paramètres ACCU et ARRA ;

- mesures à la sortie des avaloirs, pour étudier plus finement les phénomènes de stockage, de tri granulométrique et de remise en suspension des solides ;

- mesures du charriage, pour combler une lacune importante à l'heure actuelle. Les aspects métrologiques de la question ne sont pas les moindres ;

- mesures en continu des solides en suspension, notamment par l'emploi de turbidimètres, pour déterminer l'allure des pollutogrammes, en évitant les prélèvements classiques instantanés, généralement peu nombreux et trop peu représentatifs de l'évolution réelle des phénomènes ;

- mesures des dépôts et de leur évolution. Dans ce domaine également, les aspects métrologiques sont importants.

Modélisation et expérimentation in situ doivent être menées conjointement. Proposer des hypothèses a priori pour représenter les phénomènes ne prend de sens que si elles peuvent ensuite être étayées pat l'observation. Réciproquement, la multiplication des mesures permettra de proposer des hypothèses aux fondements plus sûrs et davantage représentatifs de la réalité.

Face à la grande variabilité des phénomènes observés en réseau, il est également important, grâce à la mesure, de pouvoir distinguer ce qui relève des lois générales et ce qui relève du caractère particulier de chaque réseau.

En effet, la diversité des approches et des concepts utilisés dans les modèles actuels de transport solide (Huber 1986), et la diversité des résultats qui en découle (Jacobi 1990), témoignent en définitive du caractère encore très empirique et relatif de nos connaissances sur le transport solide en réseau d'assainissement : «Concernant la formation des dépôts et le transport solide [en réseau d'assainissement] en milieu urbain, leur simulation est très difficile. [...] De nombreuses personnes ont cherché des solutions au transport des sédiments, mais il n'y a pas de résultats définitifs; il reste beaucoup de 
travail à faire [dans ce domaine]. » (Wayne C. Huber 1991, communication personnelle). Le modèle HYPOCRAS s'inscrit ainsi comme une contribution supplémentaire à ce domaine de recherche encore très ouvert.

\section{Remerciements}

Le modèle HYPOCRAS a été élaboré dans le cadre d'une collaboration avec l'Institut de mécanique des fluides de Strasbourg et l'Ecole nationale des ingénieurs des travaux ruraux et des techniques sanitaires. L'auteur remercie également R. M. Ashley du Dundee Institute of Technology pour les données mises à notre disposition.

\section{Références bibliographiques}

AlLEY W. M. (1981). - Estimation of imprevious area washoff parameters. Water Resources Research, 1981, vol. 17, $\mathrm{n}^{*} 4$, pp. 1161-1166, 12 ref.

Alley W. M., SMrth P. E. (1981), - Estimation of accumulation parameters for urban runoff quality modeling. Water Resources Research, 1981, vol. 17, $\mathrm{n}^{\circ} 6$, pp. 1657 1664,13 ref.

Ashley R. M., Coghlan B. P., Jefferies C. (1989). - The quality of sewage flows and sediment in Dundee. $2^{e}$ Wageningen Conference, CHOIIAWPRC/IAC, September $1989,8 \mathrm{p}$.

Ashley R. M., Coghlan B. P., Crabtree R. W. (1990). Sewer sediments. Their occurrence, movement and polluting potential. Scottish branch meeting, Institution of Water and Environmental Management, January 1990, 26 p., 16 ref.

Balades J. D., Bourgogne P., Bachoc A., Madiec H., Faup G.-M. (1991). - Un moyen de lutte contre la pollution des rejets urbains de temps de pluie. Les solutions compensatoires. TSM, 1991, $\mathrm{n}^{\circ} 12$, pp. 583-592, 10 ref.

Bertrand-KRAJEWSKı J. L. (1991a). - Modélisation des débits et du transport solide en réseau d'assainissement. Etude bibliographique. Rapport ENITRTS/IMF Strasbourg/Lyonnaise des Eaux-Dumez, Strasbourg, avril 1991, 207 p., 309 ref.

Bertrand-Krajewskı J. L. (1991b). - Mantes-la-Ville : données expérimentales numérisées pluie - débit - solides en suspension (d'après un rapport LROP). Rapport ENITRTS, Strasbourg, octobre 1991, 54 p.

Bertrand-Krajewskı J. L. (1992a). - Dundee Perth Road: données expérimentales pluie - débit - solides en suspension 1988-1989. Rapport ENITRTS, Strasbourg, mars 1992, 45 p., 4 ref. (non publié).

Bertrand-KRAJEWSKı J. L. (1992b). - Modélisation conceptuelle du transport solide en réseau d'assainissement unitaire. Thèse de doctorat de l'Université Louis Pasteur, Strasbourg, avril 1992, 205 p., 93 ref.

Bertrand-Krajewski J. L., Bellefleur D. (1991). Entzheim : données expérimentales pluie - débit - solides en suspension 1987-1988. Rapport ENITRTS, Strasbourg, octobre 1991, 267 p., 6 ref.

BRIAT P. (1989). - Transport solide en réseau d'assainissement. Inventaire et étude comparative des modèles. Document Lyonnaise des Eaux R/D n 97952210 , juin 1989.
Chebbo G., Musquere P., Bachoc A. (1990). - Solides transférés dans les réseaux d'assainissement. Caractéristiques hydrodynamiques et charges polluantes. Rapport IMF, Toulouse, 1990, 7 p., 18 ref.

DESBORDES M. (1974). - Réflexions sur les méthodes de calcul des réseaux urbains d'assainissement pluvial. Thèse USTL, Montpellier, 1974, 224 p., 107 ref.

HUBER W. C. (1986). - Deterministic modeling of urban runoff quality. Urban Runoff Pollution, Nato ASI series, vol. G 10, Springer Verlag, Berlin, 1986, pp. 167-242, 179 ref.

IAWPRC (1989). - Urban discharges and receiving water quality impacts. Advances in Water Pollution Control, Editor J. B. Ellis, IAWPRC, London, 1989, 190 p.

JACOBI D. (1990). - Evaluation of pollutant-load-calculationmethods by measurements. Comparison and evaluation of the methods. $5^{e}$ Int. Conf. on Urban Storm Drainage, Osaka, 1990, pp. 371-376, 4 ref.

JeWEll T. K., Adrian D. D. (1978), - SWMM stormwater pollutant washoff functions. Journal of the Environmental Engineering Division, 1978, vol. 104, n 5 , pp. 10361040, 7 ref.

Jovanovic S. (1986). - Hydrologic approaches in urban drainage system modelling. Urban Drainage Modeling, Dubrovnik, 1986, Pergamon Press, pp. 185-208, 50 ref.

LAPLACE D. (1991). - Dynamique du dépôt en collecteur d'assainissement. Thèse de doctorat de l'Institut National Polytechnique, Toulouse, 1991, 202 p.

Laveuf L., Philippe J. P., Ranchet J. (1980). - Bassin versant urbanisé de Mantes-la-Ville. Etude de la pollution véhiculée par les eaux de ruissellement en réseau unitaire. Document LROP, FAER 1.08.06.7/FAER 1.67.11.7, Trappes, 1980, 45 p. + annexes, 6 ref.

MARR G. (1976). - Analyse und Simulation des Niederschlagsabflusses in städtischen Gebieten. Der Niederschlagsabfluss in städtischen Einzugsgebieten, Technische Universität München, Heft 11, 1976, 110 p.

MOFFA P. E. (1990). - Control and treatment of combined sewer overflows. Environmental Engineering Series, Editor P. E. Moffa, Van Nostrand Reinhold, New York, $1990,226 \mathrm{p}$.

NiEMCZYNOWiCZ J. (1988). - The rainfall movement. A valuable complement to short-term rainfall data. Journal of Hydrology, 1988, vol. 104, pp. 311-326, 32 ref.

Schilling W. (1983). - Der Einfluss raümlich variabler Nierderschläge auf Kanalisationsabflüsse. 13e Abwassertechnisches Seminar, Technische Universität München, Heft 44, 1983, pp. 55-83, 13 ref.

ThiBault S. (1981), - Sciences et techniques de l'assainissement. Tome 1 : hydrologie urbaine. INSA, Laboratoire méthodes, Lyon, 1981, 142 p., 72 ref.

WAGENINGEN (1986), - Urban storm water quality and effects upon receiving waters. Proceedings of the $1^{e}$ Wageningen Conference, EWPCA/TNO-CHO, The Hague, October 1986.

WAGENINGEN (1989). - Urban storm water quality and ecological effects upon receiving waters. Proceedings of the $2^{e}$ Wageningen Conference, EWPCA/TNO-CHO, The Hague, September 1989.

WIUFF R. (1985). - Transport of suspended material in open and submerged streams. Journal of Hydraulic Engineering, 1985 , vol. $111, \mathrm{n}^{\circ} 5$, pp. 774-792, 20 ref.

WiUfF R. (1991). - Communication personnelle. Septembre 1991. 